\title{
A New Ghost-Node Method for Linking Different Models with Varied Grid Refinement
}

Jesse Dickinson', Scott C. James ${ }^{2}$, Steffen Mehl ${ }^{3}$, Mary C. Hill ${ }^{3}$, Stan Leake ${ }^{1}$, George A. Zyvoloski ${ }^{4}$, Guy Romer ${ }^{5}$, and Al-Aziz Eddebbarh ${ }^{6}$

'U.S. Geological Survey, 520 N Park Suite 221 Tucson, Arizona

jdickins@usgs.gov

${ }^{2}$ Sandia National Laboratories, Albuquerque, New Mexico

${ }^{3}$ U.S. Geological Survey, Boulder, Colorado

${ }^{4}$ Los Alamos National Laboratory, Los Alamos, New Mexico

${ }^{5}$ HSI_Geotrans, Westminster, Colorado

${ }^{6}$ Los Alamos National Laboratory, Las Vegas, Nevada

\section{Abstract}

A flexible, robust method for linking grids of locally refined models that may be constructed using different types of numerical methods is needed to address a variety of hydrologic problems. This work outlines and tests a new ghost-node model-linking method based on the iterative method of Mehl and Hill (2002, 2004). It is applicable to steady-state solutions for ground-water flow. Tests are presented for a homogeneous two-dimensional system that facilitates clear analysis of typical problems. The coupled grids are simulated using the finitedifference and finite-element models MODFLOW and FEHM. Results indicate that when the grids are matched spatially so that nodes and control volume boundaries are aligned, the new coupling technique has approximately twice the error as coupling using two MODFLOW models. When the grids are non-matching, model accuracy is slightly increased over matching grid cases. Overall, results indicate that the ghost-node technique is a viable means to accurately couple distinct models.

\section{Methods}

Model coupling is based on the procedure developed by Mehl and Hill $(2002 ; 2004)$ and has ten steps: 1) simulate the parent model for the entire domain; 2) interpolate parent heads onto child boundary (ghost) nodes; 3) relax the heads if not on the first iteration; 4) calculate the conductance between a ghost node and its corresponding child node; 5) simulate the child model subject to the relaxed heads from the parent model; 6) distribute child boundary fluxes across parent cell faces; 7) relax the fluxes if not on the first iteration; 8) check head and flux changes for convergence - convergence is achieved when the changes of heads and fluxes between successive iterations are within specified tolerances; 9) if not converged, solve the parent model subject to the relaxed fluxes from Step 7-parent cells within the domain of the child model are inactive; and, 10) go to Step 2 until convergence is achieved.

In this work, the parent model is constructed using the finite-difference code, MODFLOW-2000 (Harbaugh et al., 1990), and the child model is constructed using finitevolume code, FEHM (Zyvoloski et al., 1997). Both MODFLOW and FEHM have a specified 
hydraulic conductivity (or permeability) for a control volume that surrounds nodes at which hydraulic heads are calculated.

The accuracy of the grid coupling technique for matching and non-matching grids was tested using a two-dimensional idealized test case. Simulated heads and flows within the parent and child models are evaluated on the basis of errors, which are defined as the differences between simulated values and "true" values, which are calculated using an analytic solution.

\section{Ghost Node Method of Linking Models}

The links between the parent and child models - specified heads to solve the child model and specified flows for the parent model - are achieved using intermediary head and flow values at ghost nodes. As illustrated in Figure 1, ghost nodes are located within the parent model along a line that passes through cell centers of parent cells that lie along the model interface. Ghost nodes must also lie directly across the interface from the child node (the open blue circles in Figure 1). The interface between models is typically not equidistant from the ghost and child nodes. Each child node closest to the interface is connected to a ghost node within the parent model subject to the known conductance, hydraulic conductivity time the overlap length of the child cell with the parent cell divided by the distance from the child node to the ghost node. Child cells with two interface boundaries (e.g., at child model comers or when a child cell spans two parent cells), may be associated with two ghost nodes. The ghostnode method is a robust technique for linking non-matching grids, which occur when a parent cell borders a non-integer number of child cells (Figure 1b). Heads at the ghost nodes are constant-head boundary conditions for the child model where the ghost nodes become external head-dependent boundaries. Heads at the ghost nodes are calculated by interpolating heads from surrounding parent cell centers. Flows to ghost nodes are based on the calculated conductances, which are functions of the model geometries and the local hydraulic conductivity.
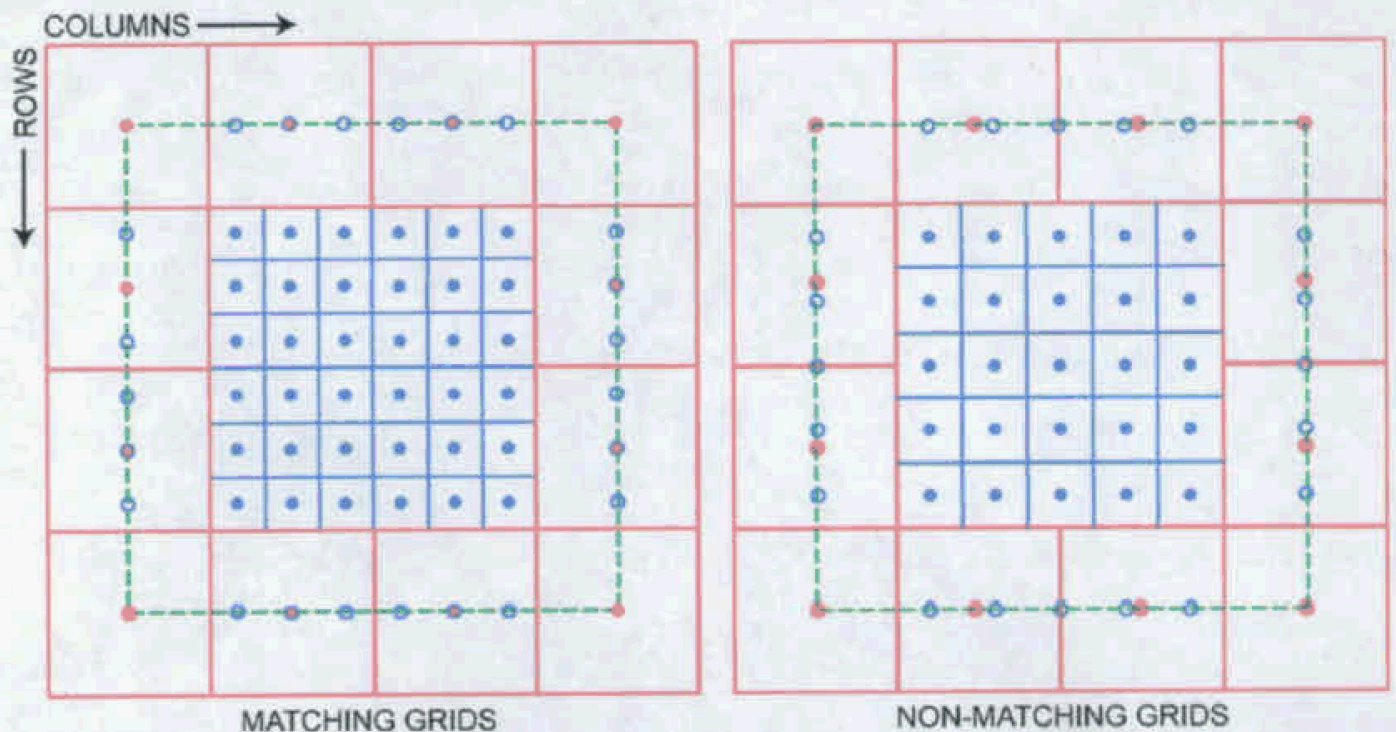

Figure 1: Two-dimensional matching (a) and non-matching (b) alignment between red parent cells and blue child cells. Parent cell centers are represented by solid red circles and child cells by solid blue circles. Ghost nodes (open blue circles) are on dashed lines passing through centers of parent cells along model interfaces and are also directly across from child cell centers. 


\section{Test Case Design}

The two-dimensional flow domain shown in Figure 2 was simulated using a steadystate parent and child model and is similar to the model configuration used by Mehl and Hill (2002). Boundary conditions in the parent model were specified heads of $10 \mathrm{~m}$ at the left side, $1 \mathrm{~m}$ along the right side, and no-flow boundaries along the top and bottom. The parent model has a $15 \times 15$ grid and overall dimensions of $1,350 \mathrm{~m}$ on all sides, which yield $90 \times 90 \mathrm{~m}^{2}$ cells. The homogeneous, isotropic, hydraulic conductivity of both models is $0.25 \mathrm{~m} / \mathrm{s}$ and the aquifer is confined. The child model is contained entirely within the parent model at the center of the flow domain. In addition, the child model has a pumping well at the center $(x=675 \mathrm{~m}$, $y=675 \mathrm{~m}$ ) that withdraws $5.5 \mathrm{~m}^{3} / \mathrm{s}$. The left and right child/parent interface boundaries are at $x=450 \mathrm{~m}$ and $x=900 \mathrm{~m}$, respectively, while the top and bottom interface boundaries are at $y=900 \mathrm{~m}$ and $y=450 \mathrm{~m}$, respectively. Different test cases have child-grid resolutions that yield both matching and non-matching grids at the interface. The 18 child model grid resolutions tested ranged from $13 \times 13$ to $47 \times 47$, which produced refinement ratios that ranged from 2.6:1 to $9.4: 1$, four of which result in matching grids: $3,5,7$, and 9:1. These simple test cases were used to evaluate simulation errors for model coupling with matching and nonmatching grids.

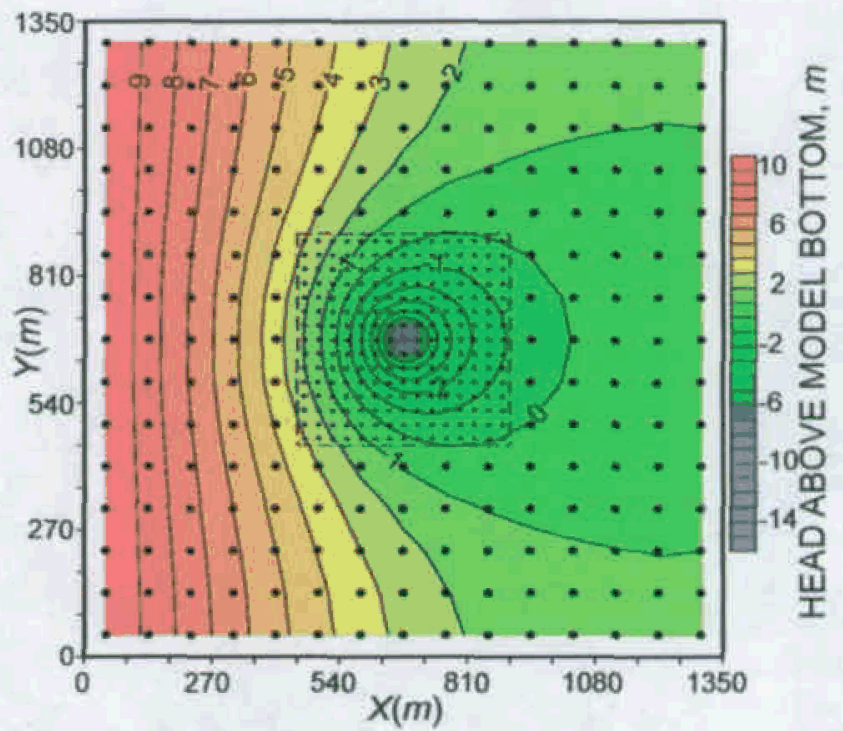

Figure 2 Head contours and observation locations for the two dimensional test case having 3:1 refinement.

\section{Results}

Simulated heads and flows for the coupled two-dimensional test case are simple enough to facilitate thorough analysis of errors by comparison to the analytic solution. Head contours are symmetrical about $x=625 \mathrm{~m}$ and decrease linearly from $10 \mathrm{~m}$ to $1 \mathrm{~m}$ from right to left, and include a cone of depression around the well at the center of the model. The solution has greater resolution near the pumping well due to the refinement of the child model, which results in smoother contours near the well than would be achieved with only the coarse parent model.

Head errors are presented as the $\mathrm{L}_{1}$ norm of head error, which is the average of the absolute values of error for selected measurement locations. Error for each cell is defined as 
the difference between values obtained after model coupling and the analytic solution. Total parent error includes differences for all cells outside of the child area, while the total child error included values from all control volumes in the child model except for the control volume containing the well.

Figure 3 indicates that the $\mathrm{L}_{1}$ norm error of both the parent and child models decreases for increased grid refinements from about $3.65 \times 10^{-3} \mathrm{~m}$ in the parent model for the $3: 1$ refinement ratio, to about $3.30 \times 10^{-3} \mathrm{~m}$ for a $9.2: 1$ refinement ratio. The error in the child model decreases from $1.92 \times 10^{-2} \mathrm{~m}$ for a $2.6: 1$ refinement ratio to about $1.25 \times 10^{-2} \mathrm{~m}$ for a 9.2:1 refinement ratio. The $\mathrm{L}_{1}$ norm error for the child model is larger than the parent error because the head solution within the child domain is more complex because the pumping well yields steep head gradients. Both the child and parent model errors decrease for increased refinement ratios because the improved accuracy of the child model benefits the solution to the parent model through application of more accurate boundary conditions. In addition, matching child models (integer number of child cells per parent cell) have increased $L_{1}$ norm errors compared to neighboring refinements. This is a result of non-matching grids having more child cells in connection with the parent cells, which results in boundary condition information being passed to the parent model from a greater spatial extent. Errors are about twice those reported by Mehl and Hill (2002) for linking two MODFLOW models.

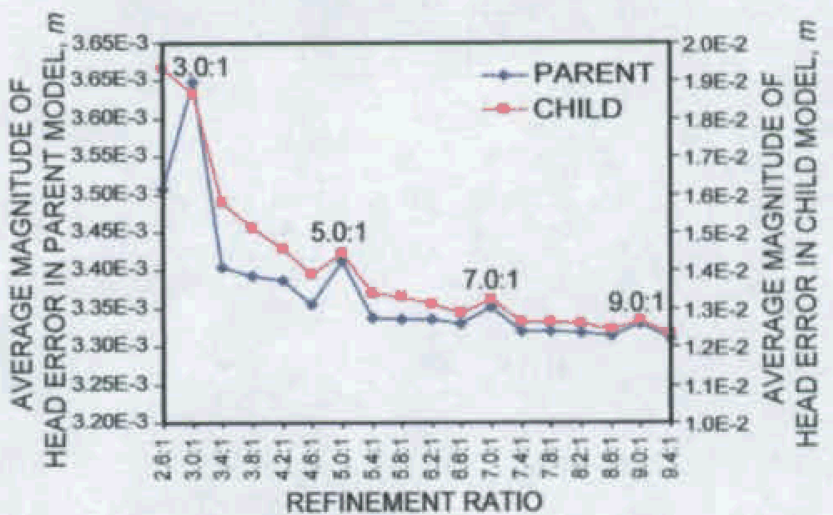

Figure 3: Flead errors of the parent and child models for two-dimensional test cases.

\section{Conclusions}

The iterative grid-coupling method requires that the parent and child model communicate boundary condition data to improve the other's solution. It is considered successful because the overall error is less than if only the regional model was used to simulate flow in the child model's domain. Errors introduced through the coupling technique are manageable and demonstrate that the methods provide a valuable new tool for estimating ground-water flow using models developed with varied levels of grid refinement. Finally, this work shows improved accuracy for non-matching grids and proves that numerical methods can be mixed across model boundaries.

\section{Acknowledgments}

This work was supported by the Yucca Mountain Site Characterization Office as part of the Civilian Radioactive Waste Management Program, which is managed by the U.S. Department of Encrgy, Yucca Mountain Site Characterization Project. Sandia National Laboratories is a multiprogram laboratory 
operated by Sandia Corporation, a Lockheed Martin Company, for the United States Department of Energy under Contract DE-AC04-94AL85000.

\section{References}

Harbaugh, A. W., E. R. Banta, M. C. Hill, and M. G. McDonald, 1990, MODFLOW-2000, The U.S. Geological Survey modular ground-water model-User guide to modularization concepts and the ground-water flow process. U.S. Geological Survey Open-File Report 90-392.

Mehl, S., and M. C. Hill, 2002, Development and evaluation of a local grid refinement method for block-centered finite-difference ground-water models using shared nodes. Adv. Water Resour., 25, 497-511.

Mehl, S., and M. C. Hill, 2004, Three-dimensional local grid refinement for block-centered finitedifference groundwater models using iteratively coupled shared nodes: a new method of interpolation and analysis of errors. Adv. Water Resour., 27, 899-912.

Zyvoloski, G. A., B. A. Robinson, Z. V. Dash, and L. L. Trease, 1997, Summary of the models and methods for the FEHM application: A finite-element heat- and mass-transfer code. Los Alamos National Laboratory Report LA-13306-MS. 